\title{
Predictors for rTMS response in chronic tinnitus
}

\section{Astrid Lehner ${ }^{1 *}$, Martin Schecklmann ${ }^{1}$, Michael Landgrebe ${ }^{1}$, Peter M. Kreuzer ${ }^{1}$, Timm B. Poeppl' Elmar Frank ${ }^{1}$, Veronika Vielsmeier ${ }^{2}$, Tobias Kleinjung ${ }^{2,3}$, Rainer Rupprecht ${ }^{1}$ and Berthold Langguth ${ }^{1}$}

\author{
Department of Psychiatry and Psychotherapy, University of Regensburg, Regensburg, Germany \\ ${ }^{2}$ Department of Otorhinolaryngology, University of Regensburg, Regensburg, Germany \\ ${ }^{3}$ Department of Otorhinolaryngology, University of Zuerich, Zuerich, Switzerland
}

\section{Edited by: \\ Jos J. Eggermont, University of Calgary, Canada}

Reviewed by:

Jos J. Eggermont, University of Calgary, Canada

Robert Folmer, Portland VA Medical Center, USA

${ }^{*}$ Correspondence:

Astrid Lehner, Department of Psychiatry and Psychotherapy, University of Regensburg,

Universitaetsstraße 84,

93053 Regensburg, Germany. e-mail: astrid.lehner@medbo.de
Background: Repetitive transcranial magnetic stimulation (rTMS) has been studied as a treatment option for chronic tinnitus for almost 10 years now. Although most of these studies have demonstrated beneficial effects, treatment results show high interindividual variability and yet, little is known about predictors for treatment response. Methods: Data from 538 patients with chronic tinnitus were analyzed. Patients received either low-frequency rTMS over the left temporal cortex $(n=345,1 \mathrm{~Hz}, 110 \%$ motor threshold, 2000 stimuli/day) or combined temporal and frontal stimulation ( $n=193,110 \%$ motor threshold, 2000 stimuli at $20 \mathrm{~Hz}$ over left dorsolateral prefrontal cortex plus 2000 stimuli at $1 \mathrm{~Hz}$ over temporal cortex). Numerous demographic, clinical, and audiological variables as well as different tinnitus characteristics were analyzed as potential predictors for treatment outcome, which was defined as change in the tinnitus questionnaire (TQ) score. Results: Both stimulation protocols resulted in a significant decrease of TQ scores. Effect sizes were small, however. In the group receiving combined treatment, patients with comorbid temporomandibular complaints benefited more from rTMS than patients without those complaints. In addition, patients with higher TQ scores at baseline had more pronounced TQ reductions than patients with low TQ baseline scores. Also, patients who had already improved from screening to baseline benefited less than patients without initial improvement. Conclusions: The results from this large sample demonstrate that rTMS shows only small but clinically significant effects in the treatment of chronic tinnitus. There are no good demographic or clinical predictors for treatment outcome.

\section{INTRODUCTION}

Subjective tinnitus is defined as a perception of sound that is not linked to an internal or external sound source. It is considered a common symptom affecting about $10-15 \%$ of adults (Hoffman and Reed, 2004) some of which are considerably impaired in their everyday lives. Chronic tinnitus is often accompanied by hearing loss, comorbid depression, sleeping problems, anxiety, and psychological stress (Halford and Anderson, 1991; Langguth, 2011). Tinnitus sufferers are considered a heterogeneous group of patients which might be divisible into several subgroups with different underlying pathophysiologies and thus benefiting from different treatment options (Landgrebe et al., 2010). Various criteria for subtyping have been proposed based both on clinical experience (Levine et al., 2008; Lindblad et al., 2011) and on empirical data (Tyler et al., 2008; Vielsmeier et al., 2011). However, it still remains a major challenge to identify useful criteria for identifying clinically relevant subtypes.

Studies using positron emission tomography (PET) and functional magnetic resonance imaging (fMRI) indicate altered neuronal activity in the central auditory system in patients with chronic tinnitus (for a review see Lanting et al., 2009). These alterations supposedly result from reorganization processes in the central nervous system that occurs as a consequence of abnormal auditory input (Eggermont, 2005). Furthermore, altered activity has also been found in non-auditory areas such as the frontal cortex or the amygdalohippocampal area (for a review see Adjamian et al., 2009). As repetitive transcranial magnetic stimulation (rTMS) is able to modify cortical excitability, it has been introduced as a new treatment option for chronic tinnitus. rTMS is a non-invasive technique applying magnetic fields for the purpose of modulating neural activity. These magnetic fields are produced by a pulsed electrical current flowing through a coil which is placed on the scalp. The magnetic fields pass through the skull and induce an electrical current in the underlying cortical neurons. If applied repetitively, long lasting changes in the excitability of directly stimulated cortical neurons as well as in functionally connected areas can be obtained. It depends on stimulation parameters if those changes act in an inhibitory or excitatory way (Ridding and Rothwell, 2007). Studies on the motor cortex revealed that low-frequency rTMS $(\leq 1 \mathrm{~Hz})$ inhibits neural activity whereas high-frequency rTMS $(\geq 5 \mathrm{~Hz})$ increases cortical excitability (Fitzgerald et al., 2006). As rTMS turned out to be effective in other hyperexcitability disorders like auditory hallucinations (Hoffman and Cavus, 2002), several studies investigated the effectiveness of low-frequency rTMS over auditory cortical areas in patients with chronic tinnitus (Kleinjung et al., 2005; 
Plewnia et al., 2007b; Smith et al., 2007; Khedr et al., 2008). Recently, the frontal cortex was examined as an additional target area as well (Kleinjung et al., 2008; Kreuzer et al., 2011). While the majority of those studies reported beneficial effects of rTMS on tinnitus severity, treatment outcomes varied highly across patients (Langguth et al., 2008a; Frank et al., 2010; Plewnia, 2011). It would, therefore, be of high clinical relevance to find out, which patient characteristics are predictive for treatment outcome as it would then be possible to offer rTMS more systematically to those patients who will most likely show positive response. Moreover, the mechanisms by which rTMS exerts beneficial effects on tinnitus are still incompletely understood (Langguth et al., 2008a; Mennemeier et al., 2011). More detailed knowledge about clinical and demographic characteristics of treatment responders may also shed light on the neurobiological mechanisms of rTMS in the treatment of tinnitus.

Some previous studies which examined the effect of rTMS on chronic tinnitus already reported an influence of clinical characteristics on treatment outcome. Tinnitus duration was found to have an effect on treatment response in studies using single sessions (De Ridder et al., 2005; Plewnia et al., 2007a) and repeated sessions of rTMS (Kleinjung et al., 2007; Khedr et al., 2008, 2010) showing that patients with shorter tinnitus duration improve more than patients with longer tinnitus duration. Some studies also indicate that patients with normal hearing develop better treatment response than patients with hearing loss (Fregni et al., 2006; Kleinjung et al., 2007) and that patients with left or bilateral tinnitus benefit more from left-sided rTMS than patients with right-sided tinnitus (Frank et al., 2010). Still, these results are debatable as there are just as many studies suggesting that tinnitus duration (Folmer et al., 2006; Rossi et al., 2007; Burger et al., 2011) or tinnitus laterality (Kleinjung et al., 2007; Rossi et al., 2007; Khedr et al., 2008) do not have any influence on treatment outcome, or that rTMS contralateral to the tinnitus side is most efficient (Khedr et al., 2010). In addition to these diverging results, most of the mentioned studies investigated only small samples and examined only the influence of demographical data, tinnitus side, and tinnitus duration on treatment outcome. Although there are studies which have taken additional characteristics into account (Kleinjung et al., 2007; Frank et al., 2010; Burger et al., 2011), there are many variables left which have not been considered yet. The aim of the current study is, therefore, to identify predictors for treatment outcome, including a larger amount of clinical and demographic variables as potential predictors. In order to identify the possible existence of different predictors for treatment with temporal and for combined frontal plus temporal rTMS, both treatment protocols were examined separately. The analysis has been conducted on pooled data stored in the database of the tinnitus research initiative (TRI) (Landgrebe et al., 2010), providing large sample sizes and thus allowing generalization of results.

\section{MATERIALS AND METHODS SUBJECTS}

Taken as a whole, data from 538 patients with chronic tinnitus were analyzed. 345 (248 men, 97 women; mean age $50.14 \pm$ 13.10 years) patients were treated with left temporal rTMS. The remaining 193 patients (135 men and 58 women, mean age $51.12 \pm 11.91$ years) received a combined frontal and temporal treatment. rTMS was either done in the course of different clinical trials (Kleinjung et al., 2005, 2008, 2009b, 2011; Langguth et al., 2006b, 2008b) or as compassionate use treatment between 2003 and January 2011. As the studies of Kleinjung et al. (2009b, 2011) revealed no enhancing effect of Levodopa or Bupropion on rTMS outcome, data of those studies were included in the current analysis. All participants were treated at the Tinnitus Center at the University of Regensburg, Germany and gave written informed consent after comprehensive explanation of the procedures. Patient data was kept confidential throughout all analyses, which have been approved by the local ethics committee. Patients with a history of epilepsy, cardiac pacemakers or other contraindications to TMS were excluded from treatment.

\section{CLINICAL ASSESSMENT AND OUTCOME MEASUREMENT}

Demographical and clinical characteristics were assessed by using the Tinnitus Sample Case History Questionnaire (Langguth et al., 2007a). Characteristics of both patient groups are given in Table 1. As not every variable was available for every patient, the table provides sample sizes for each variable separately. For calculation of the hearing level [dB HL], all thresholds measured in pure-tone audiogram from $125 \mathrm{~Hz}$ to $8 \mathrm{kHz}$ and from both sides were averaged. If hearing was too bad to assess a threshold, the value was set to $110 \mathrm{~dB}$. Tinnitus pitch was defined as the geometric mean of the lower and upper bound frequency $[\mathrm{Hz}]$ measured during the audiological examination.

Assessment of treatment effects was performed using standardized procedures as established in the TRI database (Landgrebe et al., 2010). This follows the consensus for patient assessment and outcome measurement approved by tinnitus experts from many countries during an international tinnitus conference in 2006 (Langguth et al., 2007a).

\section{REPETITIVE TRANSCRANIAL MAGNETIC STIMULATION}

Patients were treated with rTMS on 10 consecutive working days, receiving one of two possible treatment protocols. Either lowfrequency rTMS was applied over the left temporal cortex $(1 \mathrm{~Hz}$, 2000 stimuli/day) or a combined stimulation over the left temporal (1 Hz, 2000 stimuli/day) and left dorsolateral prefrontal cortex $(20 \mathrm{~Hz}, 40$ trains with 50 stimuli and an intertrain interval of $25 \mathrm{~s}$ ) was performed. As both protocols differ substantially with respect to stimulation sites and number of stimuli, data from the $1 \mathrm{~Hz}$ and $20+1 \mathrm{~Hz}$ treatment were analyzed separately. For both protocols, stimulation intensity was set at $110 \%$ of the individual resting motor threshold but never higher than $60 \%$ of maximal stimulator output. Motor threshold was defined as the minimal intensity sufficient to produce motor-evoked potentials of at least $50 \mu \mathrm{V}$ in the left thenar muscle in five out of 10 trials. Localization of the stimulated areas was either done with a neuronavigational system or by using a standard procedure based on the 10-20 system (Langguth et al., 2006b). As there is no evidence for neuronavigation being superior to the 10-20 system (Langguth et al., 2010), data were pooled without taking the localization method into account. For all patients, a Medtronic system with a figure-of-eight coil (90 mm outer diameter; Alpine 
Table 1 | Demographical data and clinical characteristics for both treatment groups.

\begin{tabular}{|c|c|c|c|c|}
\hline & $n$ & Temporal rTMS & $n$ & Frontal + Temporal rTMS \\
\hline \multirow[t]{2}{*}{ Gender } & 345 & $\mathrm{M}(72 \%)$ & 193 & $\mathrm{M}(70 \%)$ \\
\hline & & $F(28 \%)$ & & $F(30 \%)$ \\
\hline Age (years) & 345 & $50.14 \pm 13.10$ & 193 & $51.12 \pm 11.91$ \\
\hline \multirow[t]{3}{*}{ Handedness } & 334 & Right (86\%) & 186 & Right (79\%) \\
\hline & & Left $(6 \%)$ & & Left $(9 \%)$ \\
\hline & & Both Sides (8\%) & & Both Sides (12\%) \\
\hline Hearingthreshold (dB) & 224 & $20.19 \pm 12.68$ & 157 & $21.26 \pm 13.98$ \\
\hline \multirow[t]{3}{*}{ Hearing loss (left/right) } & 205 & 0-20 dB (21\%/23\%) & $146 / 148$ & $0-20 \mathrm{~dB}(23 \% / 24 \%)$ \\
\hline & & $21-60$ dB $(62 \% / 56 \%)$ & & $21-60$ dB (57\%/52\%) \\
\hline & & $>60 \mathrm{~dB}(17 \% / 21 \%)$ & & $>60 \mathrm{~dB}(20 \% / 24 \%)$ \\
\hline BDI & 221 & $9.88 \pm 7.98$ & 181 & $13.67 \pm 8.65$ \\
\hline Motor threshold & 345 & $43.14 \pm 8.50$ & 192 & $42.52 \pm 8.11$ \\
\hline TQ baseline & 345 & $38.49 \pm 17.60$ & 193 & $45.25 \pm 17.84$ \\
\hline TQ difference (baseline - screening) & 285 & $-1.99 \pm 9.48$ & 166 & $-1.43 \pm 9.58$ \\
\hline \multirow[t]{6}{*}{ Tinnitus laterality } & 335 & Right (14\%) & 188 & Right (11\%) \\
\hline & & Left (18\%) & & Left $(15 \%)$ \\
\hline & & Both ears worse left (16\%) & & Both ears worse left (21\%) \\
\hline & & Both ears worse right (16\%) & & Both ears worse right (18\%) \\
\hline & & Both ears equally (29\%) & & Both ears equally (24\%) \\
\hline & & Inside the head (7\%) & & Inside the head (11\%) \\
\hline Tinnitus duration (years) & 328 & $7.75 \pm 7.43$ & 181 & $7.62 \pm 8.73$ \\
\hline Tinnitus pitch & 104 & $6780.62 \pm 3662.23$ & 97 & $6712.60 \pm 3484.62$ \\
\hline \multirow[t]{4}{*}{ Tinnitus pitch (patient rating) } & 205 & Very high frequency (22\%) & 176 & Very high frequency (29\%) \\
\hline & & High frequency (60\%) & & High frequency (57\%) \\
\hline & & Medium frequency (16\%) & & Medium frequency (13\%) \\
\hline & & Low frequency $(2 \%)$ & & Low frequency (1\%) \\
\hline \multirow[t]{4}{*}{ Tinnitus quality } & 202 & Tone $(67 \%)$ & 173 & Tone (57\%) \\
\hline & & Noise $(8 \%)$ & & Noise (14\%) \\
\hline & & Crickets (19\%) & & Crickets $(19 \%)$ \\
\hline & & Other $(6 \%)$ & & Other $(10 \%)$ \\
\hline \multirow[t]{7}{*}{ Event-related to tinnitus onset } & 206 & Loud blast of sound (4\%) & 163 & Loud blast of sound ( $5 \%)$ \\
\hline & & Whiplash (0\%) & & Whiplash (2\%) \\
\hline & & Change in hearing $(14 \%)$ & & Change in hearing $(6 \%)$ \\
\hline & & Stress $(19 \%)$ & & Stress $(22 \%)$ \\
\hline & & Head trauma $(0 \%)$ & & Head trauma (1\%) \\
\hline & & Others (37\%) & & Others (33\%) \\
\hline & & More than one event (26\%) & & More than one event (31\%) \\
\hline \multirow[t]{3}{*}{ Pulsating tinnitus } & 181 & No $(84 \%)$ & 166 & No $(79 \%)$ \\
\hline & & Yes with heart beat (10\%) & & Yes with heart beat (9\%) \\
\hline & & Yes, different from heart beat (6\%) & & Yes, different from heart beat (12\%) \\
\hline \multirow[t]{2}{*}{ Tinnitus manifestation } & 235 & Intermittent (11\%) & 171 & Intermittent (9\%) \\
\hline & & Constant (89\%) & & Constant $(91 \%)$ \\
\hline \multirow[t]{2}{*}{ Varying Tinnitus loudness } & 189 & No $(40 \%)$ & 167 & No $(32 \%)$ \\
\hline & & Yes $(60 \%)$ & & Yes $(68 \%)$ \\
\hline \multirow[t]{3}{*}{ Tinnitus reduced by music or sounds } & 269 & No $(24 \%)$ & 170 & No $(24 \%)$ \\
\hline & & Yes $(65 \%)$ & & Yes $(62 \%)$ \\
\hline & & I don't know (11\%) & & I don't know (14\%) \\
\hline \multirow[t]{3}{*}{ Loud noise makes tinnitus worse } & 205 & No $(24 \%)$ & 169 & No $(19 \%)$ \\
\hline & & Yes $(60 \%)$ & & Yes (59\%) \\
\hline & & I don't know (16\%) & & I don't know (22\%) \\
\hline \multirow[t]{2}{*}{ Neck movement affects tinnitus } & 185 & No $(67 \%)$ & 169 & No $(63 \%)$ \\
\hline & & Yes $(33 \%)$ & & Yes (37\%) \\
\hline \multirow[t]{2}{*}{ Stress influences tinnitus } & 187 & No $(29 \%)$ & 162 & No $(72 \%)$ \\
\hline & & Yes $(71 \%)$ & & Yes $(28 \%)$ \\
\hline
\end{tabular}


Table 1 | (Continued)

\begin{tabular}{|c|c|c|c|c|}
\hline & $n$ & Temporal rTMS & $n$ & Frontal + Temporal rTMS \\
\hline \multirow[t]{2}{*}{ Suffer from headache } & 188 & No $(68 \%)$ & 168 & No $(53 \%)$ \\
\hline & & Yes (32\%) & & Yes (47\%) \\
\hline \multirow[t]{2}{*}{ Suffer from vertigo } & 326 & No $(71 \%)$ & 181 & No $(63 \%)$ \\
\hline & & Yes $(29 \%)$ & & Yes $(37 \%)$ \\
\hline \multirow[t]{2}{*}{ Temporomandibular complaints } & 287 & No $(80 \%)$ & 171 & No $(75 \%)$ \\
\hline & & Yes $(20 \%)$ & & Yes $(25 \%)$ \\
\hline \multirow[t]{2}{*}{ Suffer from neck pain } & 310 & No $(50 \%)$ & 174 & No $(37 \%)$ \\
\hline & & Yes $(50 \%)$ & & Yes $(63 \%)$ \\
\hline
\end{tabular}

Biomed, Minneapolis, MN, USA) was used with the handle of the coil pointing upwards.

\section{DATA ANALYSIS}

Statistical analysis was performed with PASW statistics 18 (SPSS Inc, Chicago, IL). All analyses were conducted separately for the $1 \mathrm{~Hz}$ and the $20+1 \mathrm{~Hz}$ group. The data analysis was based on data of the TRI Database. Data management was conducted according to the Data Handling Plan (TRI-DHP V07, 09.05.2011). Data analysis for the combined frontal and temporal group was conducted according to the Standard Operating Procedure (TRI-SA V01, 09.05.2011) thereby following a studyspecific Statistical Analysis Plan (SAP) that was written according to the SAP template (TRI-SAP 005, 26.10.2011). Data from the temporal group were analyzed analogously. All documents are to be found under http://database.tinnitusresearch.org/. Tinnitus severity was assessed at four time points using the German Version of the tinnitus questionnaire (TQ; Goebel and Hiller, 1994): before rTMS treatment ("screening," "baseline"), after the last treatment session ("day 12") and after a follow-up period of three months ("day 90"). Screening data were collected when patients visited the Tinnitus Center for the first time (generally during tinnitus consultation hours), whereas baseline data were collected immediately before treatment started. To test for changes in tinnitus severity from baseline to day 12 as well as from baseline to day 90, paired $t$-tests were used. Responder rates were calculated with responders defined as patients having improved by five points or more in the TQ score (Kleinjung et al., 2007; Frank et al., 2010). To identify predictors for treatment outcome, all variables listed in Table $\mathbf{1}$ were included as potential predictors. Besides demographical data and hearing loss, several tinnitus characteristics and selected somatic disorders like headache or neck pain were analyzed. Differences of TQ scores between baseline and day 12 as well as between baseline and day 90 were used as variables for treatment outcome. As the baseline score was used as subtrahend, negative values describe an improvement in tinnitus severity whereas positive values describe a worsening of tinnitus.

In a first step, correlations between the dependent variables and all predictors were conducted. Dependent on levels of measurement, product-moment correlations, point biserial correlations, or eta were used. $\varepsilon^{2}$ is a measure of explained variance and thus indicates, how much of the dependent variable's variance can be explained by the independent variable. In a second step, those predictors showing significant correlations with the independent variables were analyzed in a multiple regression analysis. All statistical tests were two-tailed, unadjusted for multiple comparisons and a value of $p<0.05$ was used to determine statistical significance. In correlation analyses, pair-wise deletion of missing values was applied. In regression analysis, missing values were deleted list-wisely. Data in the text are given as mean \pm standard deviation.

\section{RESULTS}

rTMS was well tolerated, no serious adverse effects were observed. Paired $t$-tests revealed a significant change of tinnitus severity at day 12 for both temporal $[T(332)=6.54, p<0.001, d=0.36]$ and combined treatment $[T(180)=3.61, p<0.001, d=0.27]$. At day 90, tinnitus severity was still significantly decreased in patients receiving combined stimulation $[T(154)=2.35, p=$ $0.012, d=0.20]$ whereas in patients receiving temporal stimulation the effect did not reach significance any more $[T(291)=$ $1.88, p=0.061, d=0.11$ ] (see Figure 1). Both groups showed similar responder rates which were stable over time: among the patients receiving temporal stimulation, $37 \%$ improved by five points or more on the TQ score at day 12 and $36 \%$ at day 90 . In the group treated with temporal plus frontal stimulation, $38 \%$ of patients were classified as responders at day 12 and $38 \%$ at day 90 .

Only some of the predictors were significantly correlated with treatment outcome and these correlations were, though statistically significant, only weak to moderate ones (see Table 2). In both groups, TQ difference from screening to baseline and treatment outcome on both day 12 and day 90 were negatively correlated. This means that those patients in which the TQ score increased from screening to baseline benefited more from treatment with rTMS than patients with improvement from screening to baseline. Furthermore, in both treatment groups, the TQ score on baseline was negatively correlated with treatment outcome on day 12 and day 90. Accordingly, patients with higher TQ scores at baseline had more pronounced TQ reductions after rTMS than patients with low TQ baseline scores. A similar result appeared for the BDI score on baseline: the higher the score on baseline, the more decrease in tinnitus severity was observed. However, this latter result is only true for treatment outcome on day 12 and only in the group receiving temporal stimulation.

In the group receiving combined treatment more investigated variables had a significant effect on treatment outcome. First, tinnitus manifestation (constant vs. intermittent) was correlated with treatment outcome on day 12 with patients with 


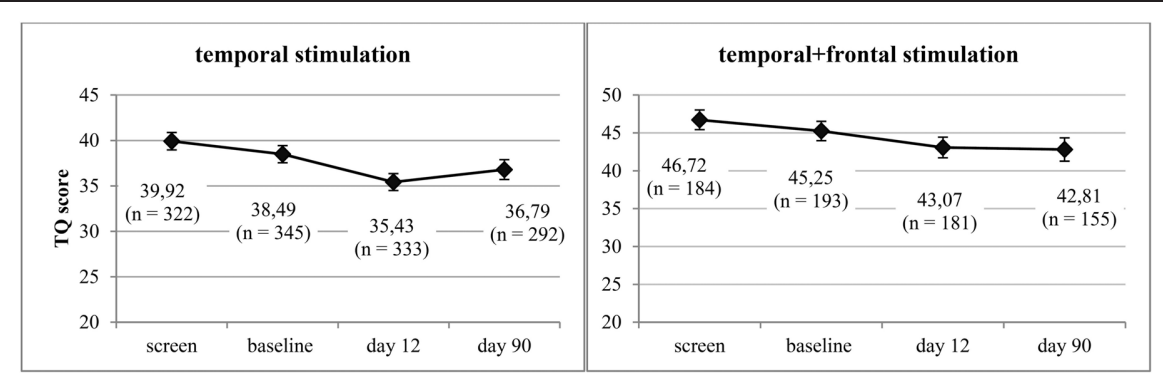

FIGURE 1 | Line chart including mean values and standard errors of TO scores before ("screen," "baseline") and after ("day 12," "day 90") rTMS treatment for both treatment groups.

Table 2 | Correlations between treatment outcome and predictors for both treatment groups.

\begin{tabular}{|c|c|c|c|c|}
\hline & \multicolumn{2}{|c|}{ Temporal rTMS } & \multicolumn{2}{|c|}{ Frontal + Temporal rTMS } \\
\hline \multicolumn{5}{|l|}{ PRODUCT MOMENT CORRELATION $r$} \\
\hline TQ difference (baseline - screen) & $-0.187 * *$ & $-0.229 * *$ & $-0.231 * *$ & $-0.183^{*}$ \\
\hline TQ baseline & $-0.313^{* *}$ & $-0.230 * *$ & $-0.189 *$ & $-0.170^{*}$ \\
\hline $\mathrm{BDI}$ & $-0.240 * *$ & -0.127 & -0.067 & -0.060 \\
\hline Hearing threshold & -0.034 & 0.007 & 0.009 & 0.086 \\
\hline Tinnitus duration (years) & 0.043 & 0.032 & -0.113 & 0.102 \\
\hline Tinnitus pitch & -0.080 & -0.146 & 0.088 & -0.013 \\
\hline \multicolumn{5}{|l|}{ POINT BISERIAL CORRELATION $r$} \\
\hline Neck movement affects tinnitus & 0.028 & -0.025 & 0.036 & 0.036 \\
\hline Stress influences tinnitus & 0.064 & 0.006 & 0.147 & 0.025 \\
\hline Suffer from headache & 0.032 & 0.087 & 0.085 & -0.015 \\
\hline Suffer from vertigo & -0.048 & -0.067 & 0.001 & 0.117 \\
\hline Temporomandibular complaints & -0.042 & -0.032 & $-0.184^{*}$ & $-0.187^{*}$ \\
\hline Suffer from neck pain & -0.099 & -0.064 & -0.045 & 0.014 \\
\hline \multicolumn{5}{|l|}{$\varepsilon^{2}$} \\
\hline Handedness & 0.003 & 0.002 & 0.004 & 0.008 \\
\hline Hearing loss (left/right) & $0.000 / 0.005$ & $0.001 / 0.011$ & $0.006 / 0.010$ & $0.010 / 0.006$ \\
\hline Tinnitus laterality & 0.006 & 0.017 & 0.025 & 0.013 \\
\hline Tinnitus pitch (patient rating) & 0.058 & 0.026 & 0.020 & 0.018 \\
\hline
\end{tabular}

$* \alpha<0.05$.

${ }^{* *} \alpha<0.001$.

constant tinnitus benefiting more from rTMS than patients with intermittent tinnitus. This effect could not be depicted for data on day 90 though. Finally, a significant influence of comorbid temporomandibular complaints on treatment outcome was found. Patients suffering from temporomandibular complaints experienced more benefit from rTMS than patients without temporomandibular complaints.

As can be seen in Table 2, $\varepsilon^{2}$ was quite low with $5.8 \%$ being the highest value. Those $5.8 \%$ are explained by "tinnitus pitch.” However, the category being responsible for this minimal 
correlation is "low pitch." Since the sample sizes of the "low pitch" groups are very small (see Table 1) the result is assumed to be an idiosyncratic effect of the small samples. The same is true for the variable "event related to onset of tinnitus." All remaining correlations did not reach statistical significance.

Regression analysis was conducted separately for both treatment groups and time points. Only variables significantly correlated with treatment outcome were included in the analyses. Consequently, each analysis contained a different number of independent variables. In all analyses, the TQ difference from screening to baseline proved to be a significant predictor for treatment outcome. For the group receiving temporal stimulation, TQ score at baseline appeared as an additional predictor for treatment outcome on day 90 but did not reach statistical significance in the remaining analyses (see Table 3). BDI score on baseline, which was only included in one regression model (temporal stimulation, day 12), was detected as a significant predictor in this model. Furthermore, in the group receiving combined stimulation, temporomandibular complaints predicted treatment outcome on both day 12 and day 90, whereas tinnitus manifestation (intermittent vs. continuous) did not serve as a relevant predictor for treatment outcome any more. The coefficients for determination in the different models range from 0.065 to 0.111 .

\section{DISCUSSION}

The current results from a large sample indicate that rTMS significantly decreases tinnitus severity in tinnitus patients. Owing to the large sample size, the rather small change in TQ scores (between 4-10\%) reaches statistical significance while the effect sizes are only small to moderate. Of course, the question arises if this small decrease in tinnitus severity can be considered not only statistically significant but also clinically relevant. It has to be taken into account that-apart from behavioral therapy (Hesser et al., 2011b)—-there are no therapeutic tools for chronic tinnitus available for which the evidence of efficacy has already been clearly provided by metaanalyses. Facing this lack of highly effective therapeutic alternatives, the observed improvement of at least 5 points in the TQ score in 36-38\% of all treated patients is a remarkable result, which represents-at least for these responders - a clinically relevant tinnitus reduction. Although the mean TQ score reduction and the effect size of
rTMS are small, it brings improvement to patients who would otherwise have no real therapeutic alternatives. The small effect sizes should, therefore, not lead to the conclusion that rTMS is not efficient enough to be examined in future studies. The small effect sizes rather point to the potential of rTMS, but also to the need for future studies to further improve treatment outcome by applying rTMS over new stimulation sites or by changing stimulation frequencies (Kleinjung and Langguth, 2009a).

Regarding the stimulation protocol, both temporal and combined stimulation resulted in a decrease of tinnitus severity on day 12 , but significant improvement on day 90 was only observable in the patients receiving combined stimulation. This result is consistent with the finding of Kleinjung et al. (2008) suggesting that combined stimulation has longer lasting effects on chronic tinnitus than temporal stimulation only.

Regarding predictors for treatment outcome, some of the characteristics analyzed were significantly correlated with treatment outcome. These correlations have to be interpreted with caution however, as they are only weak to moderate ones. Two parameters were significantly correlated to treatment outcome in both treatment groups: the change of tinnitus severity from screening to baseline and the tinnitus severity at baseline. Furthermore, in the group receiving combined stimulation, patients with comorbid temporomandibular complaints benefited more from rTMS than patients without temporomandibular complaints. Additionally, depressivity at baseline (assessed by the BDI score) was significantly correlated with treatment outcome on day 12 in patients receiving temporal stimulation. In the group receiving combined stimulation, patients with constant tinnitus showed more improvement on day 12 compared to patients with intermittent tinnitus. However, those latter results were not found for treatment outcome on day 90 indicating that neither depressivity nor tinnitus manifestation exert considerable influence on long-term effects of rTMS. This assumption is supported by regression analysis which reveals that only two of the parameters mentioned remain as significant predictors of treatment outcome: change of tinnitus severity from screening to baseline and suffering from temporomandibular disorder (for patients receiving combined treatment only).

In detail, the changing TQ score from screening to baseline is the strongest predictor for treatment outcome, reaching statistical

Table 3 | Results of multiple regression analyses for both treatment groups: standardized regression coefficients ( $\beta$ ).

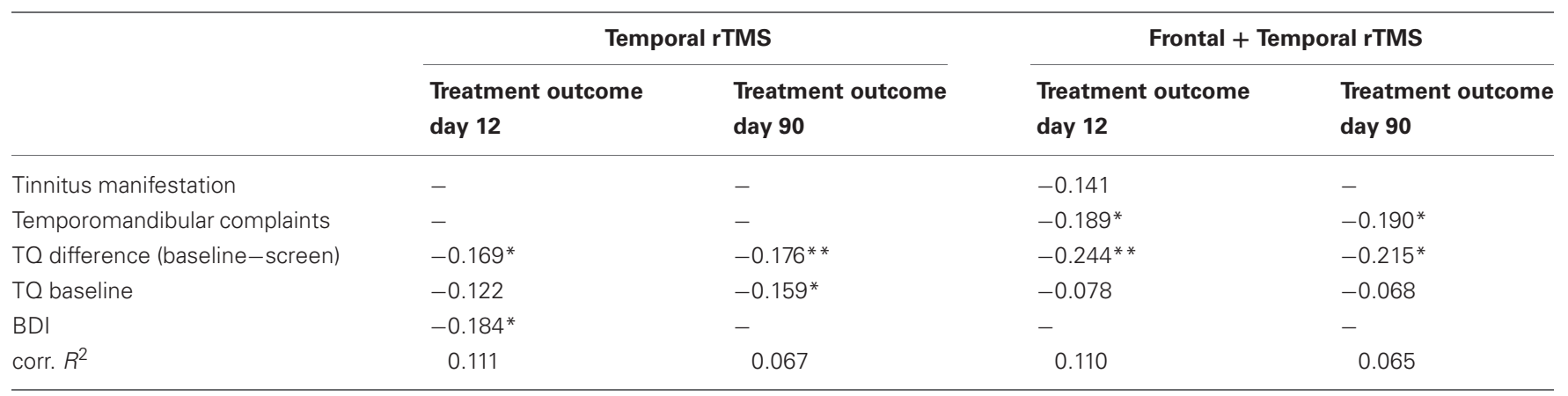

${ }^{*} \alpha<0.05$.

${ }^{* *} \alpha<0.001$. 
significance for both treatment groups and both day 12 and day 90. This robust finding is remarkable since the time interval between screening and baseline was not standardized and varied across patients. Patients worsening from screening to baseline benefited more from rTMS than patients who had improved between screening and the beginning of rTMS treatment. A similar relationship has been observed in a recent rTMS study (Kreuzer et al., 2011). Other earlier rTMS studies did not analyze whether changes of tinnitus severity before treatment start has an influence on treatment effects. Several explanations can be provided for this relative robust finding of an influence of the pre-treatment changes on treatment effects. The change between screening and baseline may reflect anticipation effects which are known from waiting list control groups (Hesser et al., 2011a). Thus, the inverse relationship between score changes before treatment and score changes during treatment could be explained by anticipation alone. Those patients, who improved already before treatment because of anticipation show less further improvement during treatment, because they have to catch up the anticipation effect first, whereas those who increase with their score between screening and baseline, have a more pronounced reduction during treatment since they realized, that their worries about the coming rTMS treatment, which might have caused the increase of the scores, were unwarranted.

From a more neurobiological approach the observed effect can be explained by the known dependency of rTMS effects on the history of synaptic activity of the stimulated brain area.

It has been shown that priming of cortical excitability with transcranial direct current stimulation modulates the effects of rTMS both over the motor cortex (Lang et al., 2004; Siebner et al., 2004) and to a lesser extent over the visual cortex (Lang et al., 2007). Based on these findings it has been suggested that effects of rTMS depend critically on the history of neuronal activity. Clinical effects can then be interpreted as normalization of pathologically increased activity (Siebner et al., 2004) providing an explanation why effects from healthy controls cannot be extrapolated on effects on patients with pathologically enhanced activity.

Thus, if we assume that the change in the tinnitus score before begin of TMS is reflected by changes of neuronal excitability in the stimulated area (van der Loo et al., 2009), then the observed inverse relation between changes before and during treatment could be explained as an rTMS induced enhancement of homeostatic mechanisms. Further studies should use neuroimaging methods for assessing neuronal activity at different time points before, during and after rTMS to further identify to which extent changes of neuronal activity before treatment beginning influence treatment effects. Moreover, it is strongly recommended that future clinical trials include multiple baseline assessments to identify the potential influence of pre-treatment dynamics on treatment effects and to rule out individual tinnitus oscillation patterns possibly interfering with treatment effects.

Although tinnitus severity at baseline was no significant predictor in the regression analysis, it correlates significantly with treatment outcome-a result that is consistent with previous studies reporting a negative relation between tinnitus severity at baseline and treatment outcome as well (Frank et al., 2010;
Burger et al., 2011). Patients with a higher TQ score on baseline showed stronger reductions in TQ scores than patients who had low scores at the beginning. As Frank et al. (2010) already conjectured, this might be partly due to the fact that treatment outcome was defined as the difference between the TQ score on baseline and day 12/day 90. This approach assumes that a reduction of five points is of comparable clinical relevance no matter if this reduction is from a score of 65 to 60 (7.7\% improvement) or from 25 to 20 (20\% improvement). This is probably not the case and it remains a matter of debate whether the actual improvement perceived by the patient is better expressed by the percentaged change of TQ scores or by the difference of TQ scores (Zeman et al., 2011). Future studies should try to bring clarity to this issue.

Apart from tinnitus severity itself, only one additional characteristic had an important influence on treatment outcome: the presence or absence of temporomandibular complaints. Patients with temporomandibular complaints benefited significantly more from combined frontal and temporal rTMS than patients without these complaints. It has been suggested that in tinnitus patients with temporomandibular complaints, abnormal somatosensoric input from the trigeminal nerve may be critically involved in the pathophysiology of tinnitus (Levine et al., 2008; Vielsmeier et al., 2011). Moreover in patients with so-called somatic tinnitus treatment interventions aiming at normalizing afferent somatic input have shown benefit (Bezerra Rocha et al., 2008; Biesinger et al., 2008).

In addition to its effect on cortical neurons rTMS always exerts an effect on peripheral nerves and muscles. Peripheral effects of rTMS have been shown to reduce pain perception (Zunhammer et al., 2011) and it has also been suggested that the peripheral effects of rTMS may be involved in tinnitus reduction after single sessions of rTMS (Vanneste et al., 2011). Thus, one could speculate that in patients with comorbid temporomandibular problems tinnitus might be especially sensitive for modulation of sensory input resulting in tinnitus reduction via peripheral effects of rTMS on the temporal muscle.

However, the ability to modulate tinnitus by jaw-, head-, or neckmovements was not related to treatment success, contradicting the explanation that patients who are especially sensitive to somatosensoric input benefit more from rTMS.

Beyond that, it is not directly evident why the effect of temporomandibular complaints is only seen in the group of patients who received combined stimulation whereas those complaints had no effect on treatment outcome in patients treated with temporal stimulation only. It seems somewhat implausible that frontal stimulation is crucial for the improvement in patients with temporomandibular complaints, since it is much more likely to reach the temporal muscle and the trigeminal nerve through temporal stimulation. Theoretically, left frontal rTMS might have had a beneficial effect on temporomandibular complaints, similar like on experimental pain (Fierro et al., 2010; Brighina et al., 2011). Unfortunately, current data contain no information about improvement of temporomandibular complaints after rTMS. It is, therefore, uncertain if improvement of temporomandibular complaints and tinnitus co-occur or if only tinnitus improves while temporomandibular complaints remain unchanged. 
Thus, replication of the current exploratory results is needed to rule out the possibility that the effect of temporomandibular complaints on treatment outcome is - though being statistically significant - a random effect only observable in the present sample of patients.

None of the remaining characteristics (see Table 1) are suitable predictors for treatment outcome. Consequently, previous results indicating that tinnitus duration (Khedr et al., 2008, 2010; Kleinjung et al., 2007), hearing loss (Fregni et al., 2006; Kleinjung et al., 2007), or tinnitus laterality (Frank et al., 2010) may predict treatment outcome are not supported. The current study used a large sample of patients with chronic tinnitus. It is, therefore, highly improbable that the failure to identify a clear set of reliable predictors is due to insufficient statistical power. Consequently, there are only three possible factors which might have caused these results: the outcome measures, the predictors or the relationship between them. This means that the TQ (and the use of its difference between two time points, respectively) might not be a suitable outcome measure. Perhaps, a rating scale for tinnitus loudness, the tinnitus handicap inventory or any other measure might have given different results. The TQ was used as it is a wellknown standard measure for tinnitus severity. Future research should analyze however, if the TQ shows enough sensitivity to change or if another outcome measure should be preferred. Furthermore, although numerous clinical variables were included as predictors for treatment outcome, it is possible that we still missed relevant characteristics. No previous study reported that rTMS might be particularly effective in patients with temporomandibular complaints-maybe just because those complaints were not measured. The same could be the case with other variables we simply did not ask for. This assumption is supported by the finding that the predictors entered into regression analyses explain only between $6.5 \%$ and $11.1 \%$ of variability in rTMS outcome. Another possibility is that clinical characteristics are less relevant for treatment outcome than neurophysiological or neuroimaging characteristics. Since the neurobiological mechanisms induced by rTMS are known to depend on the neuronal activity of the stimulated brain area, neuroimaging and electrophysiological methods may be better suitable for predicting rTMS

\section{REFERENCES}

Adjamian, P., Sereda, M., and Hall, D. A. (2009). The mechanisms of tinnitus: perspectives from human functional neuroimaging. Hear. Res. 253, 15-31.

Bezerra Rocha, C. A., Sanchez, T. G., and Tesseroli de Siqueira, J. T. (2008). Myofascial trigger point:a possible way of modulating tinnitus. Audiol. Neurootol. 13, 153-160.

Biesinger, E., Reisshauer, A., and Mazurek, B. (2008). The role of the cervical spine and the craniomandibular system in the pathogenesis of tinnitus. Somatosensory tinnitus. HNO 56, 673-677.

Brighina, F., De Tommaso, M., Giglia, F., Scalia, S., Cosentino, G., Puma,

effects (Langguth et al., 2006a, 2007b; Plewnia et al., 2007b). In this context it may be of relevance that there is only a relatively weak correlation between clinical characteristics and imaging data (Schecklmann et al., 2011a). Finally, the possibility cannot be ignored that there might be no further associations between predictors and treatment outcome-an interpretation which suggests that in many patients, it might not be rTMS specific effects which are responsible for treatment response but rather unspecific effects caused by regular physician contact and counseling. It should not be neglected, however, that several placebo-controlled studies already controlled for those effects and proved that rTMS is superior to placebo in the treatment of patients with chronic tinnitus (Kleinjung et al., 2005; Plewnia et al., 2007b; Rossi et al., 2007; Marcondes et al., 2010). Another placebo-controlled noncrossover study with an adequate sample size should try to bring further clarity to this issue (Landgrebe et al., 2008).

The most important conclusion that can be drawn from our results is that rTMS shows only small effects in the treatment of chronic tinnitus. These effects are considered clinically significant, however. There are no good demographic or clinical predictors for treatment outcome. The observed inverse relationship between changes before treatment beginning and during treatment argues for the use of multiple baseline assessments in future clinical trials. The finding that patients suffering from temporomandibular complaints benefit more from a treatment with rTMS than patients without temporomandibular complaints suggests that the effects by which rTMS exerts clinical effects may also involve the peripheral nervous system. If the clicking sounds produced during rTMS treatment are additionally taken into account (Schecklmann et al., 2011b), the influence of rTMS on chronic tinnitus might be the sum of central, somatosensoric, and auditory stimulation processes. A closer insight into this interplay may help to refine the treatment of chronic tinnitus with rTMS.

\section{ACKNOWLEDGMENTS}

We thank Helene Niebling and Sandra Pfluegl for their technical assistance in administering rTMS and collecting data. Parts of this study were funded by a grant from the Tinnitus Research Initiative (TRI).

magnetic stimulation for tinnitus: influence of tinnitus duration on stimulation parameter choice and maximal tinnitus suppression. Otol. Neurotol. 26, 616-619.

Eggermont, J. J. (2005). Tinnitus: neurobiological substrates. Drug Discov. Today 10, 1283-1290.

Fierro, B., De Tommaso, M., Giglia, F., Giglia, G., Palermo, A., and Brighina, F. (2010). Repetitive transcranial magnetic stimulation (rTMS) of the dorsolateral prefrontal cortex (DLPFC) during capsaicin-induced pain: modulatory effects on motor cortex excitability. Exp. Brain Res. 203, 31-38.

Fitzgerald, P. B., Fountain, S., and Daskalakis, Z. J. (2006). A comprehensive review of the effects of rTMS on motor cortical excitability and inhibition. Clin. Neurophysiol. 117, 2584-2596.

Folmer, R. L., Carroll, J. R., Rahim, A., Shi, Y., and Hal Martin, W. (2006). Effects of repetitive transcranial magnetic stimulation (rTMS) on chronic tinnitus. Acta Otolaryngol. Suppl. (556), 96-101.

Frank, G., Kleinjung, T., Landgrebe, M., Vielsmeier, V., Steffenhagen, C., Burger, J., Frank, E., Vollberg, G., Hajak, G., and Langguth, B. (2010). Left temporal low-frequency rTMS for the treatment of tinnitus: clinical predictors of treatment outcome-a retrospective study. Eur. J. Neurol. 17, 951-956. 
Fregni, F., Marcondes, R., Boggio, P. S., Marcolin, M. A., Rigonatti, S. P., Sanchez, T. G., Nitsche, M. A., and Pascual-Leone, A. (2006). Transient tinnitus suppression induced by repetitive transcranial magnetic stimulation and transcranial direct current stimulation. Eur. J. Neurol. 13, 996-1001.

Goebel, G., and Hiller, W. (1994). The tinnitus questionnaire. A standard instrument for grading the degree of tinnitus. Results of a multicenter study with the tinnitus questionnaire. HNO 42, 166-172.

Halford, J. B., and Anderson, S. D. (1991). Anxiety and depression in tinnitus sufferers. J. Psychosom. Res. $35,383-390$

Hesser, H., Weise, C., Rief, W., and Andersson, G. (2011a). The effect of waiting: a meta-analysis of wait-list control groups in trials for tinnitus distress. J. Psychosom. Res. 70, 378-384.

Hesser, H., Weise, C., Westin, V. Z., and Andersson, G. (2011b). A systematic review and meta-analysis of randomized controlled trials of cognitive-behavioral therapy for tinnitus distress. Clin. Psychol. Rev. 31, 545-553.

Hoffman, H. J., and Reed, G. W. (2004). "Epidemiology of tinnitus," in Tinnitus: Theory and Management, ed. J. B. Snow (Lewiston, NY: BC Decker Inc), 16-41.

Hoffman, R. E., and Cavus, I. (2002). Slow transcranial magnetic stimulation, long-term depotentiation, and brain hyperexcitability disorders. Am. J. Psychiatry 159, 1093-1102.

Khedr, E. M., Abo-Elfetoh, N., Rothwell, J. C., El-Atar, A., Sayed, E., and Khalifa, H. (2010). Contralateral versus ipsilateral rTMS of temporoparietal cortex for the treatment of chronic unilateral tinnitus: comparative study. Eur. J. Neurol. 17, 976-983.

Khedr, E. M., Rothwell, J. C., Ahmed, M. A., and El-Atar, A. (2008). Effect of daily repetitive transcranial magnetic stimulation for treatment of tinnitus: comparison of different stimulus frequencies. J. Neurol. Neurosurg. Psychiatry 79, 212-215.

Kleinjung, T., Eichhammer, P., Landgrebe, M., Sand, P., Hajak, G., Steffens, T., Strutz, J., and Langguth, B. (2008). Combined temporal and prefrontal transcranial magnetic stimulation for tinnitus treatment: a pilot study. Otolaryngol. Head Neck Surg. 138, 497-501.

Kleinjung, T., Eichhammer, P., Langguth, B., Jacob, P., Marienhagen, J., Hajak, G., Wolf, S.
R., and Strutz, J. (2005). Long-term effects of repetitive transcranial magnetic stimulation (rTMS) in patients with chronic tinnitus. Otolaryngol. Head Neck Surg. 132, 566-569.

Kleinjung, T., and Langguth, B. (2009a). Strategies for enhancement of transcranial magnetic stimulation effects in tinnitus patients. Int. Tinnitus J. 15, 154-160.

Kleinjung, T., Steffens, T., Landgrebe, M., Vielsmeier, V., Frank, E., Burger, J., Strutz, J., Hajak, G., and Langguth, B. (2011). Repetitive transcranial magnetic stimulation for tinnitus treatment: no enhancement by the dopamine and noradrenaline reuptake inhibitor bupropion. Brain Stimul. 4, 65-70.

Kleinjung, T., Steffens, T., Landgrebe, M., Vielsmeier, V., Frank, E., Hajak, G., Strutz, J., and Langguth, B. (2009b). Levodopa does not enhance the effect of low-frequency repetitive transcranial magnetic stimulation in tinnitus treatment. Otolaryngol. Head Neck Surg. 140, 92-95.

Kleinjung, T., Steffens, T., Sand, P., Murthum, T., Hajak, G., Strutz, J., Langguth, B., and Eichhammer, P. (2007). Which tinnitus patients benefit from transcranial magnetic stimulation? Otolaryngol. Head Neck Surg. 137, 589-595.

Kreuzer, P. M., Landgrebe, M., Schecklmann, M., Poeppl, T. B., Vielsmeier, V., Hajak, G., Kleinjung, T., and Langguth, B. (2011). Can temporal repetitive transcranial magnetic stimulation be enhanced by targeting affective components of tinnitus with frontal rTMS? A randomized controlled pilot trial. Front. Syst. Neurosci. 5, 88: doi: 10.3389/fnsys.2011.00088

Landgrebe, M., Binder, H., Koller, M., Eberl, Y., Kleinjung, T., Eichhammer, P., Graf, E., Hajak, G., and Langguth, B. (2008). Design of a placebo-controlled, randomized study of the efficacy of repetitive transcranial magnetic stimulation for the treatment of chronic tinnitus. BMC Psychiatry 8, 23.

Landgrebe, M., Zeman, F., Koller, M., Eberl, Y., Mohr, M., Reiter, J., Staudinger, S., Hajak, G., and Langguth, B. (2010). The Tinnitus Research Initiative (TRI) database: a new approach for delineation of tinnitus subtypes and generation of predictors for treatment outcome. BMC Med. Inform. Decis. Mak. 10, 42 .

Lang, N., Siebner, H. R., Chadaide, Z., Boros, K., Nitsche, M. A., Rothwell, J. C., Paulus, W., and Antal, A.
(2007). Bidirectional modulation of primary visual cortex excitability: a combined tDCS and rTMS study. Invest. Ophthalmol. Vis. Sci. 48, 5782-5787.

Lang, N., Siebner, H. R., Ernst, D., Nitsche, M. A., Paulus, W., Lemon, R. N., and Rothwell, J. C. (2004). Preconditioning with transcranial direct current stimulation sensitizes the motor cortex to rapid-rate transcranial magnetic stimulation and controls the direction of aftereffects. Biol. Psychiatry 56, 634-639.

Langguth, B. (2011). A review of tinnitus symptoms beyond 'ringing in the ears': a call to action. Curr. Med. Res. Opin. 27, 1635-1643.

Langguth, B., de Ridder, D. Dornhoffer, J. L., Eichhammer, P., Folmer, R. L., Frank, E., Fregni, F., Gerloff, C., Khedr, E., Kleinjung, T. Landgrebe, M., Lee, S., Lefaucheur, J. P., Londero, A., Marcondes, R., Moller, A. R., Pascual-Leone, A. Plewnia, C., Rossi, S., Sanchez, T., Sand, P., Schlee, W., Pysch, D., Steffens, T., van de Heyning, P., and Hajak, G. (2008a). Controversy: does repetitive transcranial magnetic stimulation/ transcranial direct current stimulation show efficacy in treating tinnitus patients? Brain Stimul. 1, 192-205.

Langguth, B., Eichhammer, P., Kreutzer, A., Maenner, P., Marienhagen, J., Kleinjung, T., Sand, P., and Hajak, G. (2006a). The impact of auditory cortex activity on characterizing and treating patients with chronic tinnitus-first results from a PET study. Acta Otolaryngol. Suppl. (556), 84-88.

Langguth, B., Goodey, R., Azevedo, A., Bjorne, A., Cacace, A., Crocetti, A., Del Bo, L., De Ridder, D., Diges, I., Elbert, T., Flor, H., Herraiz, C., Ganz Sanchez, T., Eichhammer, P., Figueiredo, R., Hajak, G., Kleinjung, T., Landgrebe, M., Londero, A., Lainez, M. J., Mazzoli, M., Meikle, M. B., Melcher, J., Rauschecker, J. P., Sand, P. G., Struve, M., van de Heyning, P., van Dijk, P., and Vergara, R. (2007a). Consensus for tinnitus patient assessment and treatment outcome measurement: Tinnitus Research Initiative meeting, Regensburg, July 2006. Prog. Brain Res. 166, 525-536.

Langguth, B., Kleinjung, T., Frank, E., Landgrebe, M., Sand, P., Dvorakova, J., Frick, U., Eichhammer, P., and Hajak, G. (2008b). Highfrequency priming stimulation does not enhance the effect of lowfrequency rTMS in the treatment of tinnitus. Exp. Brain Res. 184, 587-591.
Langguth, B., Kleinjung, T., Landgrebe, M., de Ridder, D., and Hajak, G. (2010). rTMS for the treatment of tinnitus: the role of neuronavigation for coil positioning. Neurophysiol. Clin. 40, 45-58.

Langguth, B., Kleinjung, T., Marienhagen, J., Binder, H., Sand, P. G., Hajak, G., and Eichhammer, P. (2007b). Transcranial magnetic stimulation for the treatment of tinnitus: effects on cortical excitability. BMC Neurosci. 8, 45.

Langguth, B., Zowe, M., Landgrebe, M., Sand, P., Kleinjung, T., Binder H., Hajak, G., and Eichhammer, P. (2006b). Transcranial magnetic stimulation for the treatment of tinnitus: a new coil positioning method and first results. Brain Topogr. 18, 241-247.

Lanting, C. P., de Kleine, E., and van Dijk, P. (2009). Neural activity underlying tinnitus generation: results from PET and fMRI. Hear. Res. 255, 1-13.

Levine, R. A., Nam, E. C., and Melcher, J. (2008). Somatosensory pulsatile tinnitus syndrome: somatic testing identifies a pulsatile tinnitus subtype that implicates the somatosensory system. Trends Amplif. 12, 242-253.

Lindblad, A. C., Hagerman, B., and Rosenhall, U. (2011). Noiseinduced tinnitus: a comparison between four clinical groups without apparent hearing loss. Noise Health 13, 423-431.

Marcondes, R. A., Sanchez, T. G., Kii, M. A., Ono, C. R., Buchpiguel, C. A., Langguth, B., and Marcolin, M. A. (2010). Repetitive transcranial magnetic stimulation improve tinnitus in normal hearing patients: a double-blind controlled, clinical and neuroimaging outcome study. Eur. J. Neurol. 17, 38-44.

Mennemeier, M., Chelette, K. C., Allen, S., Bartel, T. B., Triggs, W., Kimbrell, T., Crew, J., Munn, T., Brown, G. J., and Dornhoffer, J. (2011) Variable changes in PET activity before and after rTMS treatment for tinnitus. Laryngoscope 121, 815-822.

Plewnia, C. (2011). Brain stimulation: new vistas for the exploration and treatment of tinnitus. CNS Neurosci. Ther. 17, 449-461.

Plewnia, C., Reimold, M., Najib, A., Brehm, B., Reischl, G., Plontke, S. K., and Gerloff, C. (2007a). Dose-dependent attenuation of auditory phantom perception (tinnitus) by PET-guided repetitive transcranial magnetic stimulation. Hum. Brain Mapp. 28, 238-246. 
Plewnia, C., Reimold, M., Najib, A., Reischl, G., Plontke, S. K., and Gerloff, C. (2007b). Moderate therapeutic efficacy of positron emission tomography-navigated repetitive transcranial magnetic stimulation for chronic tinnitus: a randomised, controlled pilot study. J. Neurol. Neurosurg. Psychiatry 78, 152-156.

Ridding, M. C., and Rothwell, J. C. (2007). Is there a future for therapeutic use of transcranial magnetic stimulation? Nat. Rev. Neurosci. 8, 559-567.

Rossi, S., De Capua, A., Ulivelli, M., Bartalini, S., Falzarano, V., Filippone, G., and Passero, S. (2007). Effects of repetitive transcranial magnetic stimulation on chronic tinnitus: a randomised, crossover, double blind, placebo controlled study. J. Neurol. Neurosurg. Psychiatry 78, 857-863.

Schecklmann, M., Landgrebe, M., Poeppl, T. B., Kreuzer, P., Männer, P., Marienhagen, J., Wack, D. S., Kleinjung, T., Hajak, G., and Langguth, B. (2011a). Neural correlates of tinnitus duration and distress: a positron emission tomography study. Hum. Brain Mapp. [Epub ahead of print].
Schecklmann, M., Volberg, G., Frank, G., Hadersdorfer, J., Steffens, T., Weisz, N., Landgrebe, M., Hajak G., Greenlee, M., Classen, J., and Langguth, B. (2011b). Paired associative stimulation of the auditory system: a proof-of-principle study. PLoS One 6:e27088. doi: 10.1371/journal.pone.0027088

Siebner, H. R., Lang, N., Rizzo, V., Nitsche, M. A., Paulus, W., Lemon, R. N., and Rothwell, J. C. (2004). Preconditioning of low-frequency repetitive transcranial magnetic stimulation with transcranial direct current stimulation: evidence for homeostatic plasticity in the human motor cortex. J. Neurosci. 24, 3379-3385.

Smith, J. A., Mennemeier, M., Bartel, T., Chelette, K. C., Kimbrell, T., Triggs, W., and Dornhoffer, J. L. (2007). Repetitive transcranial magnetic stimulation for tinnitus: a pilot study. Laryngoscope 117, 529-534.

Tyler, R., Coelho, C., Tao, P., Ji, H., Noble, W., Gehringer, A., and Gogel, S. (2008). Identifying tinnitus subgroups with cluster analysis. Am. J. Audiol. 17, S176-S184.

van der Loo, E., Gais, S., Congedo, M., Vanneste, S., Plazier, M., Menovsky, T., Paul Van de Heyning, P., and
De Ridder, D. (2009). Tinnitus intensity dependent gamma oscillations of the contralateral auditory cortex. PLoS One 4:e7396. doi: 10.1371/journal.pone.0007396

Vanneste, S., Langguth, B., and De Ridder, D. (2011). Do tDCS and TMS influence tinnitus transiently via a direct cortical and indirect somatosensory modulating effect? A combined TMS-tDCS and TENS study. Brain Stimul. 4, 242-252.

Vielsmeier, V., Kleinjung, T., Strutz, J., Burgers, R., Kreuzer, P. M., and Langguth, B. (2011). Tinnitus with temporomandibular joint disorders: a specific entity of tinnitus patients? Otolaryngol. Head Neck Surg. 145, 748-752.

Zeman, F., Koller, M., Figueiredo, R. Aazevedo, A., Rates, M., Coelho, C., Kleinjung, T., de Ridder, D., Langguth, B., and Landgrebe, M. (2011). Tinnitus handicap inventory for evaluating treatment effects: which changes are clinically relevant? Otolaryngol. Head Neck Surg. $145,282-287$.

Zunhammer, M., Busch, V., Griesbach, F., Landgrebe, M., Hajak, G. and Langguth, B. (2011). rTMS over the cerebellum modulates temperature detection and pain thresholds through peripheral mechanisms. Brain Stimul. 4, 210-217.e211.

Conflict of Interest Statement: The authors declare that the research was conducted in the absence of any commercial or financial relationships that could be construed as a potential conflict of interest.

Received: 24 December 2011; paper pending published: 19 January 2012; accepted: 09 February 2012; published online: 23 February 2012.

Citation: Lehner A, Schecklmann $M$, Landgrebe M, Kreuzer PM, Poeppl TB, Frank E, Vielsmeier V, Kleinjung T, Rupprecht $R$ and Langguth B (2012) Predictors for rTMS response in chronic tinnitus. Front. Syst. Neurosci. 6:11. doi: 10.3389/fnsys.2012.00011

Copyright (c) 2012 Lehner Schecklmann, Landgrebe, Kreuzer, Poeppl, Frank, Vielsmeier, Kleinjung, Rupprecht and Langguth. This is an open-access article distributed under the terms of the Creative Commons Attribution Non Commercial License, which permits non-commercial use, distribution, and reproduction in other forums, provided the original authors and source are credited. 